\title{
Research on Power Short-term Prediction of the Photovoltaic System Based on Grey Relational Analysis and Quantum Particle Swarm Optimization
}

\author{
Qingwu Gong ${ }^{1}$, Jiazhi Lei ${ }^{1{ }^{1 *}}$, Haining Zhang ${ }^{2}$, Yang Lei ${ }^{1}$ and Si Tan ${ }^{1}$ \\ ${ }^{1}$ School of Electrical Engineering Wuhan University, Wuhan, China \\ ${ }^{2}$ Qinghai Province Key Laboratory of Photovoltaic Grid Connected Power Generation Technology, Xining, China \\ ${ }^{*}$ Corresponding author
}

\begin{abstract}
Output power of Photovoltaic generation system is influenced by temperature, humidity, solar radiation intensity and so on. The effects of three kinds of external climate conditions, including temperature, humidity, solar radiation intensity, on photovoltaic output power were anal sized in detail in this paper, and then similar days for photovoltaic power prediction were selected based on grey relational analysis. The quantum particle swarm optimization method for optimizing kernel parameters of support vector machine was immediately introduced. In line with the data of similar days and optimization parameters of kernel function, a new power short-term prediction method of the photovoltaic system based on grey relational analysis and quantum particle swarm optimization was put up in this paper. According to the data of photovoltaic output power and meteorological monitoring data of Wulan photovoltaic power station, the method mentioned is likely verified. Instances proved that this new power short-term prediction method has great advantages in terms of speed, accuracy and stability.
\end{abstract}

Keywords-photovoltaic power prediction; grey relational analysis; battery characteristics; quantum particle swarm optimization; support vector machine; wulan photovoltaic power station

\section{INTRODUCTION}

Photovoltaic generation is one of the main using ways of solar energy, and more and more attention on using solar energy is paid with rapid development of photovoltaic generation system[1].

Output power of photovoltaic generation system is influenced by external climate conditions, like temperature, humidity, solar radiation intensity and so on, and the randomness of external climate conditions result in the randomness of photovoltaic output power[2]. Since the output of the PV system is a certain randomness, it may affect the security and stability of power grid when PV system is grid connected. With large scale application of photovoltaic generation system, reasonable prediction for photovoltaic output power must be correspondingly put forward to suppress the influences of photovoltaic generation system on power grid[3].

Recently, researches of photovoltaic power prediction developed rapidly. Reference [4-5] introduced a forecasting method of time series smoothness prediction, which has few calculation and a fast calculation speed. But it is great influenced by history bizarre data, and prediction accuracy drops quickly with the increase of forecasting steps in the progress of photovoltaic power prediction. Reference [6-8] put up a artificial neural network method for photovoltaic power prediction, which has a great advantage in solving complex circumstances. But low efficiency and local optimum may be appeared during the progress of calculation, and its convergence rate is very slow. Reference [9-10] put up a forecasting method based on support vector machine, which map the original input to the high dimensional linear feature space by nonlinear kernel function. This method can deal with nonlinear problem perfectly, and achieve a smaller actual risk in prediction.

Reference [11-12] put up a multi-factor analysis method based on grey relational analysis, which analyzed the mutual influences of various factors and the contribution of each factor to the main factor. Reference [13] present a method of parameter optimization in least squares support vector machines by using of basic particle swarm optimization, which was used for short-term wind speed prediction. Reference [14] proposed the theory of quantum particle swarm optimization, which can search the global optimal solution when it is used in parameter optimization.

In this paper, specific correlation of temperature, humidity, solar radiation intensity and photovoltaic output power were analyzed by grey relational analysis method, and then similar days for photovoltaic power prediction were selected. The method of support vector machine was chosen to predict photovoltaic output power, and the quantum particle swarm optimization method for optimizing kernel parameters of support vector machine was immediately introduced. In line with the data of similar days and optimization parameters of kernel function, a new power short-term prediction method of the photovoltaic system based on grey relational analysis and quantum particle swarm optimization was put up. Finally, according to the data of photovoltaic output power and meteorological monitoring data of Wulan photovoltaic power station, this method mentioned was likely verified.

\section{INFLUENCE OF CLIMATE CONDITIONS ON Photovoltaic Output Power}

Output power of photovoltaic generation system is influenced by many factors, summarizing to two aspect 
including component performances of photovoltaic generation system and external climate conditions. Component performances of photovoltaic generation system determine the capacity and efficiency of photovoltaic generation system, which are not considered in this paper. External climate conditions, including temperature, humidity and solar radiation intensity, are the random factors that affect the randomness of photovoltaic output power[15].

Because of the incomparable of photovoltaic output power and meteorological monitoring data, these data must be normalized as followed:

$$
x_{i}^{*}=\frac{x_{i}-x_{\min }}{x_{\max }-x_{\min }}
$$

where, $x_{i}$ is the $i$ th original data, and $x_{i}^{*}$ is the ith data after normalization. $x_{\max }$ and $x_{\min }$ respectively represented the maximum value and minimum value of original data.

\section{A. Temperature}

The effects of temperature on photovoltaic output power are mainly manifested in two aspects: the one is open circuit voltage, short-circuit current and peak power of solar cell, and the other is conversion efficiency of solar cell. The correlation between temperature and photovoltaic output power is shown in Figure 1.

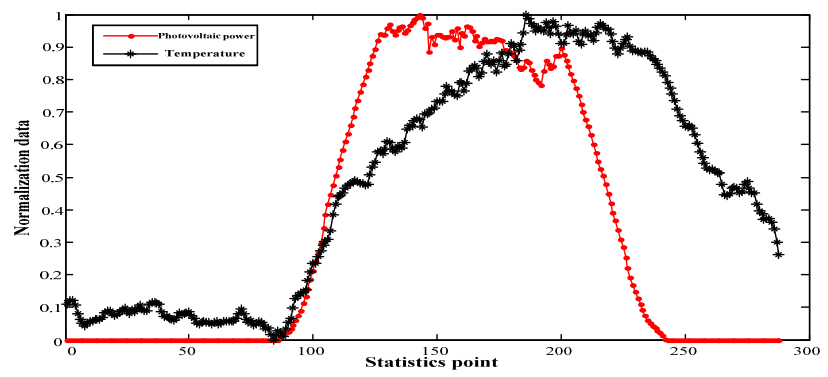

FIGURE I. CORRELATION BETWEEN TEMPERATURE AND PHOTOVOLTAIC OUTPUT POWER.

As seen in Figure 1, photovoltaic output power increases along with the increase of temperature, and photovoltaic output power reduces when temperature drops. But this tendency has a certain lag in time.

\section{B. Humidity}

Humidity can affect the solar radiation intensity. When relative humidity increases, vapor in the air increases correspondingly, which can weaken the solar radiation intensity of photovoltaic generation system, and the photovoltaic output power will cut down in the following. The correlation between humidity and photovoltaic output power is shown in Figure 2.

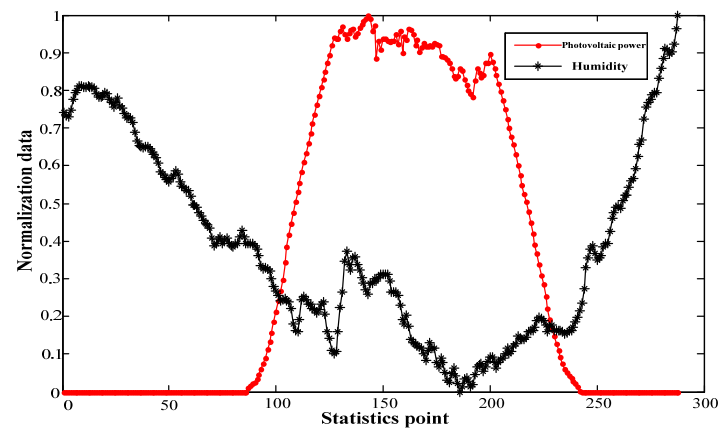

FIGURE II. CORRELATION BETWEEN HUMIDITY AND PHOTOVOLTAIC OUTPUT POWER.

As seen in Figure 2, the tendency of photovoltaic output power is substantially in contrast with humidity.

\section{Solar Radiation Intensity}

The effect of solar radiation intensity on photovoltaic output power is the most straightforward. Photovoltaic output power increases along with the increase of solar radiation intensity. The correlation between solar radiation intensity and photovoltaic output power is shown in Figure 3.

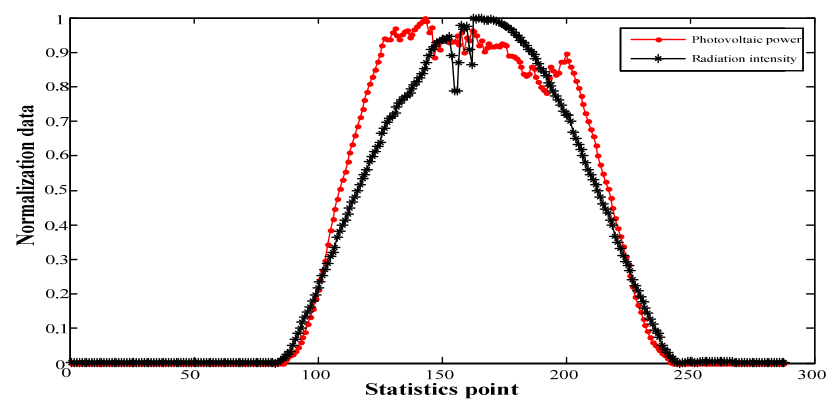

FIGURE III. CORRELATION BETWEEN SOLAR RADIATION INTENSITY AND PHOTOVOLTAIC OUTPUT POWER.

\section{Similar Days SElEcting BASED ON GREY RELATIONAL ANALYSIS}

\section{A. Grey Relational Analysis}

Grey system theory is a new method of researching less data and the uncertainty of poor information. Especially, the method based on grey relational analysis can make multi-factor analysis by comparing the similarities of curves which are described by sequence database. Before of grey relational analysis, these sequence database must be normalized by formula (1). Deng interrelatedness analysis method is adopted in this paper. Supposing the characteristic behavior sequence of the photovoltaic generation system is

$$
X_{0}(k)=\left(x_{0}(1), x_{0}(2), \cdots, x_{0}(n)\right)
$$

and behavior sequence of influencing factors is

$$
X_{i}(k)=\left(x_{i}(1), x_{i}(2), \cdots, x_{i}(n)\right)
$$


If four basic axioms of grey relational analysis are satisfied in the photovoltaic generation system[16], Deng interrelatedness coefficient is

$$
\gamma\left(x_{0}(k), x_{i}(k)\right)=\frac{\min _{i} \min _{k}\left|x_{0}(k)-x_{i}(k)\right|+\rho \max _{i} \max _{k}\left|x_{0}(k)-x_{i}(k)\right|}{\left|x_{0}(k)-x_{i}(k)\right|+\rho \max _{i} \max _{k}\left|x_{0}(k)-x_{i}(k)\right|}
$$

Correlation degree of Deng interrelatedness analysis method is

$$
\gamma\left(X_{0}, X_{i}\right)=\frac{1}{n} \sum_{k=1}^{n} \gamma\left(x_{0}(k), x_{i}(k)\right)
$$

\section{B. Similar Days Selecting}

The influences of temperature, humidity, and solar radiation intensity on photovoltaic output power were analyzed based on grey relational analysis. According to influencing degrees of various factors, corresponding weights were caught, which were used for similar days selecting in photovoltaic generation system.

In this paper, meteorological monitoring data of temperature, humidity, and solar radiation intensity were used for eigenvalues of similar days, and grey relational grades for reference sequence of individual eigenvalue were calculated by grey relational analysis.

Supposing grey relational grades of $n$ influencing factors are $\beta_{1}, \beta_{2}, \cdots, \beta_{n}$, the influencing weight of $i$ th influencing factor on photovoltaic output power is

$$
\alpha_{i}=\beta_{i} / \sum_{i=1}^{n} \beta_{i}
$$

For $i$ th influencing factor, influencing data of forecast day are used for reference data, which is recorded as $F_{0}(i)(i=1,2, \cdots, n)$. Similarly, influencing data of forecast day are used for comparison data, which is recorded as $F_{j}(i)(i=1,2, \cdots, n)$. Where, $j(j=1,2, \cdots, m)$ is the serial number of history day, and $m$ is the number of history days.

According to formula (2) and (3), grey relational grade of $i$ th influencing factor can be calculated, and the grey relational grade is denoted as $\lambda_{i}(i=1,2, \cdots, n)$. Similarly, grey relational grade of every influencing factor can be calculated individually, and similarity value of history day to forecast day is given as follows:

$$
S(j)=\sum_{i=1}^{n} \alpha_{i} \lambda_{i}
$$

In line with the magnitude of similarity values, appropriate numbers of similar days are therefore selected for photovoltaic power prediction.

\section{QUANTUM PARTICLE SWARM OPTIMIZATION FOR SUPPORT VECTOR MACHINE}

\section{A. Support Vector Machine}

After appropriate numbers of similar days were selected, support vector machine is used for photovoltaic power prediction by finding out the function law of the data of similar days.

Support vector machine(SVM) is a new machine learning method based on statistical learning theory, and it has become the research field of machine learning because of its excellent performance[17]. Actually, the mechanism of SVM is to find a optimal separating hyperplane, which maximize the blank area on both sides of the hyperplane with a great separating accuracy simultaneously. In mathematics, SVM can be expressed as[18]:

$$
\begin{gathered}
\min _{w, b} \frac{1}{2} \sum_{i=1}^{l} \sum_{j=1}^{l}\left(\alpha_{i}^{*}-\alpha_{l}\right)\left(\alpha_{j}^{*}-\alpha_{j}\right) K\left(x_{i}, x_{j}\right)+\varepsilon \sum_{j=1}^{l}\left(\alpha_{i}^{*}+\alpha_{l}\right)-\sum_{i=1}^{l} y_{i}\left(\alpha_{l}^{*}-\alpha_{l}\right) \\
\text { s.t. }\left\{\begin{array}{l}
\sum_{i=1}^{l}\left(\alpha_{i}-\alpha_{i}^{*}\right)=0 \\
0 \leq \alpha_{i} \leq C, 0 \leq \alpha_{i}^{*} \leq C, i=1,2, \cdots, l
\end{array}\right\}
\end{gathered}
$$

where, $\varepsilon$ is insensitive loss coefficient, $\alpha^{*}$ is the optimal solution, $C$ is penalty factor, and input sample

$$
T=\left\{\left(x_{i}, y_{i}\right)\right\}, x_{i} \in R^{n}, y_{i} \in\{-1,1\}, i=1,2, \cdots, l
$$

Gauss kernel function has good comprehensive properties, and is used as the kernel function $K\left(x_{i}, x_{j}\right)$.

$$
K\left(x_{i}, x_{j}\right)=\exp \left(-\frac{\left\|x_{i}-x_{j}\right\|^{2}}{\sigma^{2}}\right)
$$

According to equation (6) and (7), the parameters $(\mathrm{C}, \delta, \varepsilon)$ the key problem of SVM.

\section{B. Quantum Particle Swarm Optimization}

Particle swarm optimization, as an adaptive evolutionary calculation technology, was put up by Kennedy and Eberhart in 1995[19]. Global optimal solution of parameter optimization could be easily got by quantum particle swarm algorithm. This novel global convergent algorithm has fewer parameters, faster convergence speed as well as strong search ability for complex problems. 
In quantum space, speed and position of a particle cannot be determined simultaneously. Wave function $\mu$ is used to describe the state of the particle, and probability density function of a particle appearing at a point is acquired by solving Schrodinger equation[20]. And then, position equation of the particle at time $t$ is given as follows:

$$
X(t)=P \pm \frac{L}{2} \ln (1 / \mu)
$$

where, $\mu \in[0,1]$, and $L$ is defined as:

$$
L(t+1)=2 * \beta * \mid \text { mbest }-X(t) \mid
$$

where, mbest is defined as:

$$
\text { mbest }=\sum_{i=1}^{M} P_{i} / M=\left(\sum_{i=1}^{M} P_{i 1} / M, \cdots, \sum_{i=1}^{M} P_{i D} / M\right)
$$

where, $\beta$ is the coefficient of contraction and expansion, $M$ is the number of particles, $D$ is the space dimension of particles, $P_{i}$ is the pbest of $i$ th particle. Though equation (8) and (9), position equation of the particle at time $\mathrm{t}+1$ is given as follows:

$$
X(t+1)=P \pm \beta * \mid \text { mbest }-X(t) \mid * \ln (1 / \mu)
$$

According to equation (8)-(11), optimal coefficients $X$ can be acquired by iterative iteration with a suitable coefficient of contraction and expansion $\beta$.

In this paper, the parameters $(C, \delta, \varepsilon)$ of support vector machine were optimized by quantum particle swarm algorithm. And then, the model of support vector machine was established for photovoltaic output power prohibition based on the data of similar days.

\section{CASE STUDY}

In this section, the data of photovoltaic output power and meteorological monitoring data of Wulan photovoltaic power station of April, 2012 were used to prohibit the photovoltaic output power, which can softly verify the accuracy and stability of this short-term prediction method based on grey relational analysis and quantum particle swarm optimization.

April 28 was chosen as the forecast day, and climate similarities of April 4 to April 27 with the forecast day in the aspect of photovoltaic power prohibition can be calculated, as shown in Table I.

In line with the magnitude of similarity values, dates 25,16 , $26,15,23$, and 27 are selected as similar days for Wulan photovoltaic output power prohibition.
TABLE I. CLIMATE SIMILARITY OF HISTORY DAYS TO FORECAST DAY.

\begin{tabular}{cccc}
\hline Dates & $\begin{array}{c}\text { Grey relational } \\
\text { grade }\end{array}$ & Dates & $\begin{array}{c}\text { Grey relational } \\
\text { grade }\end{array}$ \\
\hline 25 & 0.8030 & 18 & 0.6716 \\
16 & 0.7912 & 14 & 0.6710 \\
26 & 0.7566 & 4 & 0.6624 \\
15 & 0.7549 & 8 & 0.6596 \\
23 & 0.7545 & 21 & 0.6524 \\
27 & 0.7281 & 11 & 0.6469 \\
24 & 0.7207 & 7 & 0.6423 \\
10 & 0.6979 & 20 & 0.6296 \\
17 & 0.6908 & 9 & 0.5883 \\
22 & 0.6901 & 6 & 0.5670 \\
5 & 0.6890 & 13 & 0.5469 \\
19 & 0.6804 & 12 & 0.5392 \\
\hline
\end{tabular}

\section{A. Prohibition Based on Quantum Particle Swarm} Optimization

Gauss kernel function was used as the kernel function of support vector machine for photovoltaic output power prohibition. Average relative error of prediction data was used as the value of fitness, the optimum parameters $(C, \delta, \varepsilon)$ could be acquired by quantum particle swarm optimization.

$$
C=18.9507, \sigma=0.1759, \varepsilon=0.0115
$$

With using of the optimum parameters $(C, \delta, \varepsilon)$ and support vector machine algorithm, the forecast results of photovoltaic output power were shown as Figure 4.

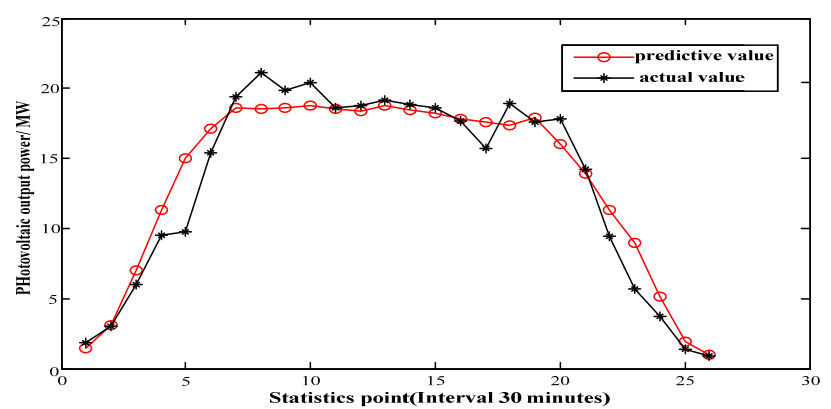

FIGURE IV. FORECAST RESULTS BASED ON QUANTUM PARTICLE SWARM OPTIMIZATION.

In Figure 4, a prediction was done per 30 minutes, and consuming time of the whole forecasting process $t=12.81 \mathrm{~s}$. As seen in Figure 4, the predictive values of photovoltaic output power were very close to the actual values. The average absolute error is $1.2053 \mathrm{MW}$, and the average relative error is $14.15 \%$.

\section{B. Prohibition Based on YSPSO and SPSO}

In line with the data of similar days, forecast results of photovoltaic output power on April 28 were also acquired based on two basic particle swarm optimization methods (YSPSO and SPSO)[22]. Forecast results based on YSPSO was shown as Figure 5 and forecast results based on SPSO was shown as Figure 6. 


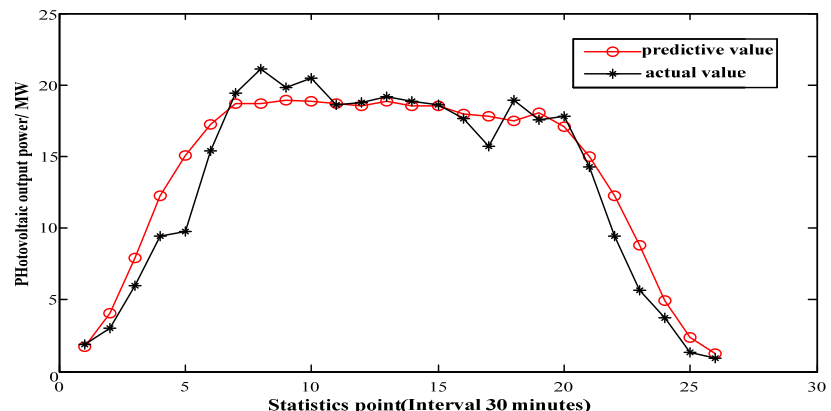

FIGURE V. FORECAST RESULTS BASED ON YSPSO.

In Figure 5, consuming time of the whole forecasting process $t=29.67 \mathrm{~s}$. The average absolute error is $1.4165 \mathrm{MW}$, and the average relative error is $16.63 \%$.

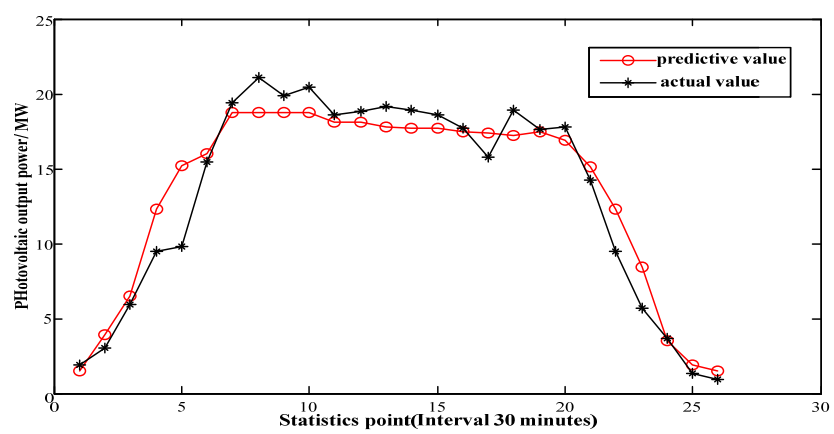

FIGURE VI. FORECAST RESULTS BASED ON SPSO.

In Figure 6, consuming time of the whole forecasting process $t=41.47 \mathrm{~s}$. The average absolute error is $1.3441 \mathrm{MW}$, and the average relative error is $15.78 \%$.

\section{Comparison}

In the aspect of prediction accuracy, average relative error of QPSO is slightly smaller than YSPSO and SPSO, as shown in Figure 4-6. On the other hand, consuming time of QPSO is greatly shorter than YSPSO and SPSO.

\section{CONCLUSION}

(1) The effects of three kinds of external climate conditions, including temperature, humidity, solar radiation intensity, on photovoltaic output power are significantly clear.

(2) This new prediction method based on grey relational analysis and quantum particle swarm optimization has faster calculation speed, higher accuracy as well as stronger stability by case verification and comparison.

\section{ACKNOWLEDGMENT}

This work was supported by The National Key Technology R\&D Program of China (2013BAA02B01) and Qinghai Province Key Laboratory of Photovoltaic Grid Connected Power Generation Technology (2014-Z-Y34A). The authors gratefully acknowledge the assistance of Qinghai electric power corporation of China in providing large amounts of data. In addition, Zhang Haining, as one of the authors, did so much researches and provided a lot of help for this paper.

\section{REFERENCES}

[1] Schaefer J C. Review of photovoltaic power plant performance and economics[J]. IEEE Transaction on Energy Conversion, 1990, (52): 232238.

[2] Ramenah, H., Tanougast, C., Cicero, L.. Toward a prediction of the photovoltaic based power production from Experimental Thermal modeling[C]. IEEE Conference on Transportation Electrification AsiaPacific, 2014: 1-4.

[3] Liserre, M., Teodorescu, R., Blaabjerg, F.. Stability of grid-connected PV inverters with large grid impedance variation[C] IEEE 35th Annual Conference on Power Electronics Specialists, 2004: 4773-4779.

[4] BACHER P, MADSEN H, NIELSEN H A. Online short-term solar power forecasting[J]. Solar Energy, 2009, 83(10): 1772-1783.

[5] Lei Shaolan, Sun Caixin, Zhou Quan. Method of Multivariate Time Series of Short-Term Load Forecasting[J]. Transactions of China Electrotechnical Society, 2005, 20(4): 62-67.

[6] Application of recurrent neural network to short-term-ahead generating power forecasting for photovoltaic system[C]// IEEE Power Engineering Society General Meeting, June 24-28, 2010, Tampa, USA: 1-6.

[7] WANG Fei, MI Zeng-qiang, SU Shi, et al. Short-term solar irradiance forecasting model based on artificial neural network using statistical feature parameters[J]. Energies, 2012, 5: 1355-1370.

[8] SULAIMAN SI, RAHMAN T K A, MUSIRIN I. Partial evolutionary ANN for output prediction of a grid-connected photovoltaic system [J]. International Journal of Computer and Electrical Engineering, 2009, 1 (1): $40-45$.

[9] Jie Shi, Wei-Jen Lee, Yongqian Liu, et al. Forecasting Power Output of Photovoltaic Systems Based on Weather Classification and Support Vector Machines[J]. IEEE Transactions on Industry Applications, 2012, 3(48): 1064-1069.

[10] Tamas K, Remus T, Marco L, et al. Evaluation of three-phase transformerless photovoltaic inverter topologies[J]. IEEE Trans on Power Electronics, 2009, 24(9): 2202-2211.

[11] Cui Jie, Dang Yao-guo. Study on affine property of some algorithms for grey relational analysis[C]// IEEE International Conference on Grey Systems and Intelligent Services, Nov. 10-12, 2009: 52-56.

[12] Shin-Hsing Chang, John H. Wu, Li Zhu. Optimizing acurad die casting process via grey relational analysis[J]. The Journal of Grey System, 1997, 9(3): 247-258

[13] ZENG Jie, ZHANG Hua. A Wind Speed Forecasting Model Based on Least Squares Support Vector Machine[J]. Power System Technology, 2009, 18(33): 144-147.

[14] Hongyuan Gao, Ming Diao. Quantum Particle Swarm Optimization for MC-CDMA Multiuser Detection[C]// International Conference on Artificial Intelligence and Computational Intelligence, Nov. 7-8, 2009: 132-136.

[15] Evangelos R, Stathis T, Carsten H. Stability and power quality issues in microgrids under weather disturbances[J]. IEEE Journal of Selected Topics in Applied Earth Observations and Remote Sensing, 2008, 1(3): 170-179.

[16] Liu Sifeng. Generalized Degree of Grey Incidence[M]. Information and Systems, Dilian DMU Publishing House, 1992: 113-116.

[17] GUO Hu-sheng, WANG Wen-jian, MEN Chang-qian. A novel learning model-kernel granular support vector machine $[\mathrm{C}] / /$ Proceeding of the eighth international conference on machine learning and cybernetic, Baoding, 2009: 930-935.

[18] SYED N, LIU H, SUNG K. Incremental learning with support vector machines[C]// International Joint Conference on Artificial Intelligence. Sweden: Morgan kaufmann publishers, 1999: 352-356.

[19] Kennedy J, Eberhart R. Particle swarm optimization [C]//Proceedings of IEEE Conference on Neural Networks. Perth, Australia: IEEE, 1995: 1942-1948.

[20] Amari S, Wu S. Improving support vector machine classifiers by modifying kernel functions[J]. Neural Networks, 1999: 783 789. 